\title{
GENERIC COHOMOLOGY FOR TWISTED GROUPS
}

\author{
BY
}

GEORGE S. AVRUNIN

\begin{abstract}
Let $G$ be a simple algebraic group defined and split over $k_{0}=F_{p}$, and let $\sigma$ be a surjective endomorphism of $G$ with finite fixed-point set $G_{\sigma}$. We give conditions under which cohomology groups of $G$ are isomorphic to cohomology groups of $G_{\sigma}$.
\end{abstract}

Let $G$ be a simple algebraic group defined and split over $k_{0}=\mathbf{F}_{p}$, and, for $q=p^{m}$, let $G(q)$ be the subgroup of $\mathbf{F}_{q}$-rational points. For a finite dimensional rational $G$-module $V$ and a nonnegative integer $e$, let $V(e)$ be the $G$-module obtained by twisting the original $G$-action on $V$ by the Frobenius endomorphism $x \rightarrow x^{\left[p^{e}\right]}$ of $G$. Cline, Parshall, Scott, and van der Kallen proved in [2] that, for sufficiently large $q$ and $e$ (depending on $V$ and $n$ ), the restriction map induces an isomorphism from the rational cohomology group $H^{n}(G, V(e))$ to $H^{n}(G(q), V(e))$. This implies that, as $q$ increases, the groups $H^{n}(G(q), V(e))$ have a stable or generic value $H_{\text {gen }}^{n}(G, V)$.

In this paper, we prove an analogous theorem with $G(q)$ replaced by $G_{\sigma}$ for a surjective endomorphism $\sigma$ of $G$ having finite fixed point set. The first section of the paper summarizes the basic results on endomorphisms of algebraic groups required for the proof. Some arithmetic facts are established in the second section, and the main theorem is proved in the third section.

The author is grateful to Leonard L. Scott for several helpful conversations, and to the referee for comments on an earlier version of this paper.

1. Endomorphisms of algebraic groups. We briefly review here some results on endomorphisms of algebraic groups which will be needed later. We refer the reader to [4] and [5] for more details.

Let $k$ be the algebraic closure of the prime field $k_{0}=F_{p}$ and let $G$ be a simple algebraic group defined and split over $k_{0}$. If $\sigma$ is a surjective rational endomorphism of $G$ having finite fixed-point set $G_{\sigma}$, $\sigma$ stabilizes a Borel subgroup $B$ and a maximal torus $T \leqslant B$. All such pairs $(B, T)$ are $G_{o}$-conjugate.

Assume $G_{\sigma}$ is finite and fix a $\sigma$-stable pair $(B, T)$. Let $\Sigma$ be the root system of $T$ in $G$, with fundamental system $\Delta \leqslant \Sigma^{+}$defined by $B$. The comorphism $\sigma^{*}$ of $\left.\sigma\right|_{T}$ determines a permutation $\rho$ of $\Sigma$ stabilizing $\Sigma^{+}$and $\Delta$, and powers $q(\alpha)$ of $p$ such that $\sigma^{*} \rho(\alpha)=q(\alpha) \alpha$. If $U_{\alpha} \leqslant G$ is the root subgroup determined by $\alpha$ and $x_{\alpha}$ : ${ }_{k}$ Add $\rightarrow U_{\alpha}$ is a $T$-equivariant isomorphism, then $\sigma x_{\alpha}(u)=x_{\rho \alpha}\left(c_{\alpha} u^{q(\alpha)}\right)$ for some

Received by the editors May 5, 1980 and, in revised form, November 12, 1980.

1980 Mathematics Subject Classification. Primary 20G10.

Key words and phrases. Cohomology of algebraic groups, finite groups of Lie type.

(C) 1981 American Mathematical Society 0002-9947/81/0000-0513/\$02.75 
$c_{\alpha} \in k$. Adjusting the isomorphisms $x_{\alpha}$ as necessary, the $c_{\alpha}$ can be taken to be \pm 1 , with $c_{\alpha}=1$ if $\alpha$ or $-\alpha$ is fundamental.

If $\rho$ is the identity, all $q(\alpha)$ are equal, $G_{o}$ is a finite Chevalley group, and the results of [2] apply. We are primarily concerned with the cases in which $\rho$ is a nontrivial permutation. These are listed below.

I. $G$ is of type $A_{n}, \rho$ is the nontrivial graph automorphism, and $q(\alpha)=p^{f}$ for all $\alpha . G_{\sigma}$ is ${ }^{2} A_{n}\left(p^{f}\right)$.

II. $G$ is of type $D_{n}, \rho$ is a graph automorphism of order 2 , and $q(\alpha)=p^{f}$ for all $\alpha$. $G_{\sigma}$ is ${ }^{2} D_{n}\left(p^{f}\right)$.

III. $G$ is of type $D_{4}, \rho$ is a graph automorphism of order $3, \operatorname{ad} q(\alpha)=p^{f}$ for all $\alpha$. $G_{\sigma}$ is ${ }^{3} D_{4}\left(p^{f}\right)$.

IV. $G$ is of type $E_{6}, \rho$ is the nontrivial graph automorphism, and $q(\alpha)=p^{f}$ for all $\alpha . G_{\text {o }}$ is ${ }^{2} E_{6}\left(p^{f}\right)$.

V. $G$ is of type $C_{2}, \rho$ interchanges long and short roots, $p=2$, and $q(\alpha)=2^{f}$ if $\alpha$ is long, while $q(\alpha)=2^{f+1}$ if $\alpha$ is short. $G_{\sigma}$ is ${ }^{2} C_{2}\left(2^{f}\right)$.

VI. $G$ is of type $F_{4}$. If the successive nodes of the Dynkin diagram are $\alpha_{1}, \alpha_{2}, \alpha_{3}$ and $\alpha_{4}$ with $\alpha_{1}$ and $\alpha_{2}$ long, $\rho$ interchanges $\alpha_{1}$ and $\alpha_{4}$ and interchanges $\alpha_{2}$ and $\alpha_{3}$. $p=2$ and $q(\alpha)$ is as in case V. $G_{\sigma}$ is ${ }^{2} F_{4}\left(2^{f}\right)$.

VII. $G$ is of type $G_{2}, \rho$ interchanges long and short roots, $p=3$, and $q(\alpha)=3^{f}$ if $\alpha$ is long, while $q(\alpha)=3^{f+1}$ if $\alpha$ is short. $G_{\sigma}$ is ${ }^{2} G_{2}\left(3^{f}\right)$.

2. Arithmetic results. In the next section, we will show that certain sufficient conditions for the restriction map $H^{n}(G, V) \rightarrow H^{n}\left(G_{\sigma}, V\right)$ to be an injection or an isomorphism reduce to conditions on particular systems of equations. Here we establish some results concerning these systems of equations. Our arguments are based on Theorem 1 below, which follows from Lemma 6.4 and the discussion at the beginning of $\$ 6$ of [2].

THEOREM 1. Let $\alpha, \beta, x$ and $d$ be integers. Suppose

$$
\sum_{j=1}^{a} p^{i_{j}}=p^{\alpha} x+d\left(p^{\beta}-1\right)
$$

with $a \leqslant n$ and $0 \leqslant i_{j}<\beta$ for each $j$. Let [ ] denote the greatest integer function. If $\alpha \geqslant[(n-1) /(p-1)]$ and $\beta \geqslant\left[\log _{p}(|x|+1)\right]+\alpha+2$, then $d=0$.

THEOREM 2. Let $e, f, x, y, c, d, d^{\prime}$ and $n$ be integers with $0 \leqslant e$ and $0<c<p^{2 f}-$ 1. Suppose

$$
\sum_{j=1}^{a} p^{i_{j}}=p^{e} x+p^{f} c+d\left(p^{2 f}-1\right)
$$

and

$$
\sum_{l=1}^{a^{\prime}} p^{k_{l}}=p \mathfrak{y}-c+d^{\prime}\left(p^{2 f}-1\right)
$$

where $0 \leqslant a, a^{\prime} \leqslant n$ and $0 \leqslant i_{j}, k_{l}<f$ for each $j, l$.

If $e \geqslant[(2 n-1) /(p-1)]$ and $f \geqslant\left[\log _{p}(\max (|x|,|y|)+1)\right]+e+2$, then $c=d$ $=d^{\prime}=0$. 
Proof. Multiplying the second equation by $p^{f}$ and adding gives

$$
\sum_{j=1}^{a} p^{i_{j}}+p^{f} \sum_{l=1}^{a^{\prime}} p^{k_{l}}=p^{3}\left(x+p^{f_{y}}\right)+\left(d+p^{f} d^{\prime}\right)\left(p^{2 f}-1\right)
$$

The hypotheses of the theorem imply that $2 f \geqslant\left[\log _{p}\left(\left|x+p^{f} y\right|+1\right)\right]+e+2$ and $e \geqslant\left[\left(a+a^{\prime}-1\right) /(p-1)\right]$. Theorem 1 then gives $d+p^{f} d^{\prime}=0$. Multiplying the resulting equation by $p^{f}$ and rewriting, we have

$$
p^{f} \sum_{j=1}^{a} p^{i_{j}}+\sum_{l=1}^{a^{\prime}} p^{k_{l}}=p^{e}\left(p^{f} x+y\right)+\left(p \mathcal{y}-\sum_{l=1}^{a^{\prime}} p^{k_{l}}\right)\left(p^{2 f}-1\right) .
$$

Another application of Theorem 1 yields $p y-\sum_{l=1}^{a^{\prime}} p^{k_{l}}=0$ from which it follows that $c=d=d^{\prime}=0$.

TheOREM 3. Let e, $f, x, y, c, d, d^{\prime}$ and $n$ be integers with $0<e$ and $0<c<p^{2 f+1}$ - 1. Suppose

$$
\sum_{j=1}^{a} p^{i_{j}}=p^{e} x+p^{f} c+d\left(p^{2 f+1}-1\right)
$$

and

$$
\sum_{l=1}^{a^{\prime}} p^{k_{l}}=p^{e} y-c+d^{\prime}\left(p^{2 f+1}-1\right)
$$

where $0<a, a^{\prime} \leqslant n$ and $0<i_{j}, k_{l} \leqslant f$ for each $j$, l. If $e \geqslant[(2 n-1) /(p-1)]$ and $f \geqslant\left[\log _{p}(\max (|x|,|y|)+1)\right]+e+2$, then $c=d=d^{\prime}=0$ and $i_{j}, k_{l}<$ for each $j$, l.

Proof. Arguing as before, we apply Theorem 1 with $\beta=2 f+1$ to obtain $\sum_{j=1}^{a} p^{i}+p^{f} \sum_{l=1}^{a^{\prime}} p^{k_{t}}=p^{e} x+p^{e+f_{y}}$. The hypothesis on $f$ implies that the right side is less than $p^{2 f}$, so each $k_{l}<f$. Then after multiplying by $p^{f}$ and rewriting as $\mathrm{n}$ the previous proof, we may apply Theorem 1 with $\beta=2 f$ to conclude that $\sum p^{k_{t}}=p^{e} y$. Then $\sum p^{i_{j}}=p^{e} x$, so $c=d=d^{\prime}=0$, and, since $\left|p^{e} x\right|<p^{f}$, each $i_{j}<f$.

Theorem 4. Let $e, f, x, y, z, c, c^{\prime}, d, d^{\prime}, d^{\prime \prime}$ and $n$ be integers with $0<e$ and $0<c$, $c^{\prime}<p^{3 f}-1$. Suppose

$$
\begin{aligned}
& \sum_{j=1}^{a} p^{i_{j}}=p^{e} x+p^{f} c+p^{2 f} c^{\prime}+d\left(p^{3 f}-1\right) \\
& \sum_{l=1}^{a^{\prime}} p^{k_{l}}=p^{e} y-c+d^{\prime}\left(p^{3 f}-1\right)
\end{aligned}
$$

and

$$
\sum_{s=1}^{a^{\prime \prime}} p^{r_{s}}=p^{e} z-c^{\prime}+d^{\prime \prime}\left(p^{3 f}-1\right)
$$

where $0 \leqslant a, a^{\prime}, a^{\prime \prime} \leqslant n$ and $0 \leqslant i_{j}, k_{l}, r_{s}<f$ for each $j, l$, s. Then if $e>$ $[(3 n-1) /(p-1)]$ and $f>\left[\log _{p}(\max (|x|,|y|,|z|)+1)\right]+e+2, c=c^{\prime}=d=d^{\prime}$ $=d^{\prime \prime}=0$. 
Proof. Combining the three equations gives

$$
\begin{aligned}
\sum_{j=1}^{a} p^{i j} & +p^{f} \sum_{l=1}^{a^{\prime}} p^{k_{l}}+p^{2 f} \sum_{s=1}^{a^{\prime \prime}} p^{r_{s}} \\
& =p^{e}\left(x+p^{f} y+p^{2 f} z\right)+\left(d+p^{f} d^{\prime}+p^{2 f} d^{\prime \prime}\right)\left(p^{3 f}-1\right) .
\end{aligned}
$$

Theorem 1 implies that $d+p^{f} d^{\prime}+p^{2 f} d^{\prime \prime}=0$. Multiplying by $p^{f}$, we have $p^{f} \sum_{j=1}^{a} p^{i_{j}}+p^{2 f} \sum_{l=1}^{a^{\prime}} p^{k_{l}}+\sum_{s=1}^{a^{\prime \prime}} p^{r_{s}}=p^{e}\left(p^{f} x+p^{2 f_{y}}+z\right)+\left(p^{e} z-\sum_{s=1}^{a^{\prime \prime}} p^{r_{s}}\right)\left(p^{3 f}-1\right)$.

This time Theorem 1 gives $p^{e} z=\sum p^{r_{s}}$, so $c^{\prime}=d^{\prime \prime}=0$. Again multiplying by $p^{f}$ and applying Theorem 1 yields $p^{e} y=\Sigma p^{k_{t}}$ and $p^{e} x=\Sigma p^{i_{j}}$, which completes the proof.

3. The main theorem. Let $G$ be a simple algebraic group defined and split over $k_{0}$ and let $\sigma$ be a surjective rational endomorphism of $G$ having finite fixed-point set $G_{\sigma}$. Fix a $\sigma$-stable Borel subgroup $B$ and maximal torus $T \leqslant B$.

Let $U$ be the unipotent subgroup of $B$ and let $P_{1}, \ldots, P_{l}$ be the orbits of $\rho$ on $\Sigma^{+}$. Renumbering the $P_{i}$ if necessary, we can find a $\sigma$-stable central series $U=U_{1}>\cdots>U_{l}>U_{l+1}=1$ with $\bar{U}_{i}=U_{i} / U_{i+1}=\Pi_{\alpha \in P_{i}} U_{\alpha}[1]$. $T_{\sigma}$ acts on the factor group $\left(U_{i}\right)_{\sigma} /\left(U_{i+1}\right)_{\sigma}$ of the central series $U_{\sigma}=\left(U_{1}\right)_{\sigma}>\cdots>\left(U_{l}\right)_{\sigma}>1$ with weight $\left.\alpha_{i}\right|_{T_{\sigma}}$. We will write $\left(\bar{U}_{i}\right)_{\sigma}$ for $\left(U_{i}\right)_{\sigma} /\left(U_{i+1}\right)_{\sigma}$.

Let $V$ be a rational $G$-module. By Theorem 2.1 of [2], $H^{n}(G, V) \cong H^{n}(B, V)$. We are interested in conditions which insure that the restriction map $H^{n}(G, V) \rightarrow$ $H^{n}\left(G_{\sigma}, V\right)$ is an isomorphism or an injection. Since the index of $B_{\sigma}$ in $G_{\sigma}$ is prime to $p$, restriction from $H^{n}\left(G_{\sigma}, V\right)$ to $H^{n}\left(B_{\sigma}, V\right)$ is always injective, and it is then easy to see that if restriction from $H^{n}(B, V)$ to $H^{n}\left(B_{\sigma}, V\right)$ is an isomorphism (or an injection), so is restriction from $H^{n}(G, V)$ to $H^{n}\left(G_{\sigma}, V\right)$.

By the spectral sequence argument of $[2, \S 5]$, restriction $H^{n}(B, V) \rightarrow H^{n}(B, V)$ will be an isomorphism for $n \leqslant m$ and an injection for $n=m+1$, if, for each weight $\lambda$ in $V$, restriction induces an isomorphism

$$
\begin{aligned}
\left(H^{s_{1}}\left(\bar{U}_{1}, k\right) \otimes \cdots\right. & \left.\otimes H^{s_{l}}\left(\bar{U}_{l}, k\right)\right)_{-\lambda} \\
& \rightarrow\left(H^{s_{1}}\left(\left(\bar{U}_{1}\right)_{\sigma}, k\right) \otimes \cdots \otimes H^{s_{l}}\left(\left(\bar{U}_{l}\right)_{\sigma}, k\right)\right)_{\left(-\lambda \mid I_{\sigma}\right)}
\end{aligned}
$$

whenever $s_{1}+\cdots+s_{l} \leqslant m$.

The cohomology $\operatorname{ring} H^{*}\left(\bar{U}_{i}, k\right)$ is isomorphic to $\bigotimes_{\alpha \in P_{i}} H^{*}\left(U_{\alpha}, k\right)$. Let $V_{\alpha}$ be the $k$-vector space with basis $a\left(-p^{i} \alpha\right), i=0,1,2, \ldots$, and let $T$ act on $V_{\alpha}$ by $\operatorname{ta}(\mu)=\mu(t) a(\mu)$. Similarly let $W_{\alpha}$ be the $k$-vector space with basis $b\left(-p^{i} \alpha\right)$, $i=1,2, \ldots$, and $T$-action given by $t b(\mu)=\mu(t) b(\mu)$. For a vector space $V$, let $S(V)$ and $\Lambda(V)$ denote the symmetric and exterior algebras of $V$ respectively. Then [2, Theorem 4.1] as graded $T$-algebras

$$
H^{*}\left(U_{\alpha}, k\right) \simeq \begin{cases}S\left(V_{\alpha}\right) & \text { if } p=2 \\ \Lambda\left(V_{\alpha}\right) \otimes S\left(W_{\alpha}\right) & \text { if } p \neq 2\end{cases}
$$

where $V_{\alpha}$ has degree 1 and $W_{\alpha}$ has degree 2 . 
Choose a representative $\alpha_{i} \in P_{i}$ for each orbit, taking $\alpha_{i}$ long if there are two root lengths, and put $q_{i}=\prod_{\alpha \in P_{i}} q(\alpha)$. Let $V_{\alpha_{i}}\left(q_{i}\right)$ be the $k$-vector space with basis $\bar{a}\left(-p^{j} \alpha_{i}\right), j=0,1, \ldots, \log _{p} q_{i}-1$, and let $W_{\alpha_{i}}\left(q_{i}\right)$ be the $k$-vector space with basis $\bar{b}\left(-p^{j} \alpha_{i}\right), j=1,2, \ldots, \log _{p} q_{i}$. We define $T_{\sigma}$-actions on $V_{\alpha_{i}}\left(q_{i}\right)$ and $W_{\alpha_{i}}\left(q_{i}\right)$ in the same way as the $T$-actions on $V_{\alpha}$ and $W_{\alpha}$ above. Exactly as in [2, Theorem 4.1], we see that, as graded $T_{\sigma}$-algebras

$$
H^{*}\left(\left(\bar{U}_{i}\right)_{o}, k\right) \simeq \begin{cases}S\left(V_{\alpha_{i}}\left(q_{i}\right)\right) & \text { if } p=2 \\ \Lambda\left(V_{\alpha_{i}}\left(q_{i}\right)\right) \otimes S\left(W_{\alpha_{i}}\left(q_{i}\right)\right) & \text { if } p \neq 2\end{cases}
$$

where $V_{\alpha_{i}}\left(q_{i}\right)$ has degree 1 and $W_{\alpha_{i}}\left(q_{i}\right)$ has degree 2 .

Let $f=f(\sigma)=\min _{\alpha \in \Sigma^{+}} \log _{p} q(\alpha)$, as in $\S 1$. The proof of the preceding isomorphism shows that, for $p^{j}<p^{f}$, the restriction map sends $a\left(-p^{j} \alpha_{i}\right)$ to $\bar{a}\left(-p^{j} \alpha_{i}\right)$. If $\rho \alpha_{i} \neq \alpha_{i}$, the restriction of $a\left(-p^{j} \rho \alpha_{i}\right)$ is $\bar{a}\left(-p^{j+f} \alpha_{i}\right)$, and, if $\rho^{2} \alpha_{i} \neq \alpha_{i}$, the restriction of $a\left(-p^{j} \rho^{2} \alpha_{i}\right)$ is $\bar{a}\left(-p^{j+2 f} \alpha_{i}\right)$. Similarly, if $1<p^{j} \leqslant p^{f}, b\left(-p^{j} \alpha_{i}\right)$ restricts to $\bar{b}\left(-p^{j} \alpha_{i}\right)$, when $\rho \alpha_{i} \neq \alpha_{i} b\left(-p^{j} \rho \alpha_{i}\right)$ restricts to $\bar{b}\left(-p^{j+f} \alpha_{i}\right)$, and when $\rho^{2} \alpha_{i} \neq \alpha_{i} b\left(-p^{j} \rho^{2} \alpha_{i}\right)$ restricts to $\bar{b}\left(-p^{j+2 f} \alpha_{i}\right)$.

Then we have the following sufficient conditions for the map of (3.1) to be an injection or an isomorphism.

Injectivity condition for $p=2$. In every equation $\sum_{j=1}^{n} p^{i_{j}} \alpha_{j}=\lambda$, with $0 \leqslant i_{j}$ and $\alpha_{j} \in \Sigma^{+}$for each $j$, we have each $i_{j}<f$.

Isomorphism condition for $p=2$. If $\left.\sum_{j=1}^{n} p^{i} \alpha_{j}\right|_{T_{o}}=\left.\lambda\right|_{T_{o}}$ with $0 \leqslant i_{j}<f\left(0 \leqslant i_{j} \leqslant f\right.$ if there are two root lengths) and $\alpha_{j} \in \Sigma^{+}$for each $j$, then $\sum_{j=1}^{n} p^{i j} \alpha_{j}=\lambda$.

Injectivity condition for $p \neq 2$. In every equation $\sum_{j=1}^{n_{1}} p^{i_{j}} \alpha_{j}+\sum_{l=1}^{n_{2}} p^{k_{l}} \alpha_{l}=\lambda$, with $0 \leqslant i_{j}, 1 \leqslant k_{l}, \alpha_{j}, \alpha_{l} \in \Sigma^{+}$for each $j$ and $l$, and $n_{1}+2 n_{2}=n$, we have $i_{j}<f$ and $k_{l} \leqslant f$ for each $j$ and $l$.

Isomorphism condition for $p \neq 2$. If $\left.\sum_{j=1}^{n_{1}} p^{i_{j}} \alpha_{j}\right|_{T_{o}}+\left.\sum_{l=1}^{n_{2}} p^{k_{1}} \alpha_{l}\right|_{T_{o}}=\left.\lambda\right|_{T_{o}}$ with $0 \leqslant i_{j}$ $<f, 0<k_{l} \leqslant f\left(0 \leqslant i_{j} \leqslant f, 0<k_{l} \leqslant f+1\right.$ if there are two root lengths), $\alpha_{j}$, $\alpha_{l} \in \Sigma^{+}$for each $j$ and $l$, and $n_{1}+2 n_{2}=n$, the $\sum_{j=1}^{n_{1}} p^{i} \alpha_{j}+\sum_{k=1}^{n_{2}} p^{k_{l}} \alpha_{l}=\lambda$.

Let $\omega=\Sigma_{\delta \in \Delta} n_{\delta} \delta$ be the maximal root and put $b=\max n_{\delta}$. For $\zeta$ in the root lattice $Q$, write $\zeta=\Sigma_{\delta \in \Delta} m_{\delta} \delta$ and put $b(\zeta)=\max \left|m_{\zeta}\right|$. For $\lambda$ in the weight lattice $\Lambda$, let $t(\lambda)$ be the order of $\lambda$ in $\Lambda / Q$, with $t_{p}(\lambda)$ the $p$-part of $t(\lambda)$, and let $t$ be the exponent of $\Lambda / Q$. We write $\bar{\lambda}=t \lambda$.

For integers $b, n$ and $e$, let

$$
e_{\sigma}(n)= \begin{cases}{[(n-1) /(p-1)]} & \text { if } \rho=1 \\ {[(2 n-1) /(p-1)]} & \text { if } \rho \neq 1 \text { and } G_{\sigma} \text { is not }{ }^{3} D_{4} \\ {[(3 n-1) /(p-1)]} & \text { if } G_{\sigma} \text { is }{ }^{3} D_{4}\end{cases}
$$

and $f(b, e)=\left[\log _{p}(|b|+1)\right]+e+2$.

THEOREM 5. Let $V$ be a finite dimensional rational $G$-module and $m$ a nonnegative integer. Let $e$ be a nonnegative integer with $e \geqslant e_{\sigma}(m b t)$ and suppose $f=f(\sigma)>$ $f(b(\bar{\lambda}), e)$ for all weights $\lambda$ in $V$. If $p \neq 2$, assume further that

$$
e \geqslant(m+p-1) b t_{p}(\lambda) /(p-1) .
$$

Then the restriction map $H^{n}(G, V(e)) \rightarrow H^{n}\left(G_{o}, V(e)\right)$ is an isomorphism for $n<m$ and injection for $n=m+1$. 
Proof. First assume $p=2$.

To check injectivity, assume $\sum_{j=1}^{m+1} p^{i} \alpha_{i}=p^{e} \lambda$. If some $i_{j} \geqslant f$, multiply both sides of the equation by $t$. Examining the coefficients of $\alpha_{i}$ on both sides, we get $t p^{f} \leqslant p^{e} b(\bar{\lambda})<p^{f}$, a contradiction.

To check the isomorphism condition assume that $\left.\sum_{j=1}^{n} p^{i_{j}} \alpha_{j}\right|_{T_{o}}=\left.p^{e} \lambda\right|_{T_{o}}$, with $0 \leqslant i_{j}<f\left(0 \leqslant i_{j} \leqslant f\right.$ if there are two root lengths) and $\alpha_{i} \in \Sigma^{+}$for each $j$. Multiplying both sides by $t$, we have $\left.\sum_{v=1}^{t n} p^{u_{v}} \alpha_{v}\right|_{T_{o}}=\left.p^{e} \bar{\lambda}\right|_{T_{o}}$ where $0 \leqslant u_{v}<f$ $\left(0 \leqslant u_{v} \leqslant f\right.$ if there are two roots lengths) and $\alpha_{v} \in \Sigma^{+}$for each $v$.

Consider the map on the root lattice given by restricting a weight from $T$ to $T_{\sigma} . t$ times the kernel of this map is contained in the group generated by the elements $p^{f} \alpha_{i}-\rho \alpha_{i}$ (which may be $\left(p^{f}-1\right) \alpha_{i}$ ), and $p^{2 f} \alpha_{i}-p^{2} \alpha_{i}$ if $G_{\sigma}={ }^{3} D_{4}$ for the orbit representatives $\alpha_{i}$ in $\Delta$, and the elements $p^{2 f} \delta_{i}-\delta_{i}$ if $G_{\sigma}$ is ${ }^{2} A_{n},{ }^{2} D_{n},{ }^{2} E_{6}, p^{3 f} \delta_{i}-\delta_{i}$ if $G_{\sigma}$ is ${ }^{3} D_{4}$, and $p^{2 f+1} \delta_{i}-\delta_{i}$ if $G_{\sigma}$ is ${ }^{2} C_{2}$ or ${ }^{2} F_{4}$, where $\delta_{i}$ ranges over $\Delta$ (cf. [3, §4a]).

Thus $\sum_{v=1}^{t n} p^{\mu_{v}} \alpha_{v}$ and $p^{e} \bar{\lambda}$ differ by an element of this group. Considering the coefficients of elements of a $\rho$-orbit on $\Delta$ leads to systems of equations of the forms discussed $\S 2$, and the hypotheses of the theorem imply that the appropriate conditions on $e$ and $f$ in the theorems of that section are satisfied. Then the results of $\S 2$ imply that $\sum_{v=1}^{t n} p^{u_{v}} \alpha_{v}=p^{e} \bar{\lambda}$, so $\sum_{j=1}^{n} p^{i_{j}} \alpha_{j}=p^{e} \lambda$.

Now suppose $p$ is odd. The injectivity condition is proved exactly as when $p=2$. For the isomorphism condition, suppose $\left.\sum_{j=1}^{n_{1}} p^{i_{j}} \alpha_{j}\right|_{T_{o}}+\left.\sum_{l=1}^{n_{2}} p^{k_{1}} \alpha_{l}\right|_{T_{o}}=\left.p^{e} \lambda\right|_{T_{o}}$ with $0 \leqslant i_{j}<f\left(0 \leqslant i_{j} \leqslant f\right.$ if there are two root lengths $), 0<k_{l} \leqslant f\left(0<k_{l} \leqslant f+1\right.$ if there are two root lengths) and $\alpha_{j}, \alpha_{l} \in \Sigma^{+}$for each $j$ and $l$, and $n_{1}+2 n_{2} \leqslant m$. We want to reduce this to the arithmetic problems of $\S 2$, so we have to eliminate those $p^{k_{l}}=f\left(=f+1\right.$ in the case of two root lengths). But $\left.p^{f} \alpha\right|_{T_{o}}=\left.(\rho \alpha)\right|_{T_{o}}$ if $\Sigma$ has one root length and $\left.p^{f+1}\right|_{T_{o}}=\left.p(\rho \alpha)\right|_{T_{o}}$ if $\Sigma$ has two root lengths and $\alpha$ is long, while $\left.p^{f+1} \alpha\right|_{T_{o}}=\left.(\rho \alpha)\right|_{T_{o}}$ if there are two root lengths $\alpha$ is short.

Making the appropriate replacements of $p^{k_{1}} \alpha_{l}$ by terms with the same restriction to $T_{\sigma}$ we get $\left.\sum_{j=1}^{n_{1}} p^{i_{j}} \alpha_{j}\right|_{T_{o}}+\left.\sum_{l=1}^{n_{2}} p^{k_{l}^{\prime}} \alpha_{l}^{\prime}\right|_{T_{o}}=\left.p^{e} \lambda\right|_{T_{o}}$ with $0 \leqslant k_{l}^{\prime}<f\left(0 \leqslant k_{l}^{\prime}<f+1\right.$ if there are two root lengths). The argument in the $p=2$ case shows that $\sum_{j=1}^{n_{1}} p^{i_{j}} \alpha_{j}+\sum_{l=1}^{n_{2}} p^{k_{i}^{\prime}} \alpha_{l}^{\prime}=p^{e} \lambda$.

Now suppose $\alpha_{l}^{\prime} \neq \alpha_{l}$ for some $l$, and multiply the preceding equation by $t_{p}(\lambda)$. Subtract a simple root $\delta$ appearing in $\alpha_{l}^{\prime}$ from both sides, and consider the coefficients of $\delta$. On the left side we have at most $(m-1) b t_{p}(\lambda)$ terms of the form $p^{k} \delta$, and one term of the form $t_{p}(\lambda) m_{\delta}\left(\alpha_{l}^{\prime}\right)-1$ if there is one root length or there are two root lengths and $\alpha_{l}^{\prime}$ is long, or of the form $t_{p}(\lambda) p m_{\delta}\left(\alpha_{l}^{\prime}\right)-1$ if $\alpha_{l}^{\prime}$ is short. On the right side, the coefficient of $\delta$ is $p^{e} m_{\delta}\left(t_{p}(\lambda) \lambda\right)-1$, where $m_{\delta}\left(t_{p}(\lambda) \lambda\right)>1$.

Then the sum of the $p$-adic digits of the coefficient of $\delta$ on the left is at most $(m-1) b t_{p}(\lambda)+p t_{p}(\lambda) b-1=(m+p-1) b t_{p}(\lambda)-1$. The sum of the $p$-adic digits of the coefficient on the right is at least $e(p-1) \geqslant(m+p-1) b t_{p}(\lambda)$, which is a contradiction.

Thus $\alpha_{l}^{\prime}=\alpha_{l}$ for all $l$, and the proof is complete.

The theorem says that if $e$ is sufficiently large (with respect to $n$ ) and $f(\sigma)$ is sufficiently large (with respect to $V$ and $e$ ), then $H^{n}(G, V(e)) \simeq H^{n}\left(G_{\sigma}, V(e)\right.$ ). Since $H^{n}\left(G_{\sigma}, V\right) \simeq H^{n}\left(G_{\sigma}, V(e)\right)$, this implies that for large values of $f(\sigma)$, $H^{n}\left(G_{\sigma}, v\right)$ has a stable or generic value $H_{\text {gen }}^{n}(G, V)$. 
It is also natural to consider twisting the module $V$ by $\sigma$. If $r: G \rightarrow G L(V)$ is the morphism of algebraic groups associated with $V$, let $V(\sigma)$ denote the module with associated morphism $r \circ \sigma$. Since the weights of $T$ in $V(\sigma)$ are all divisible by $p^{f(\sigma)}$, the arguments above imply that, for $f(\sigma)$ sufficiently large with respect to $n$, $H^{n}(G, V(\sigma)) \simeq H_{\text {gen }}^{n}(G, V)$. Thus, the generic cohomology group $H_{\text {gen }}^{n}(G, V)$ may be computed as $H^{n}\left(G_{\sigma}, V\right)$ if $f(\sigma)$ is sufficiently large with respect to $n$ and $V$, or as $H^{n}(G, V(\sigma))$ if $f(\sigma)$ is sufficiently large with respect to $n$.

\section{REFERENCES}

1. G. S. Avrunin, A vanishing theorem for second degree cohomology, J. Algebra 53 (1978), 382-388.

2. E. Cline, B. Parshall, L. Scott and W. van der Kallen, Rational and generic cohomology, Invent. Math. 39 (1977), 143-169.

3. W. Jones and B. Parshall, On the l-cohomology of finite groups of Lie type, Proc. Conf. Finite Groups (W. R. Scott and F. Gross, eds.), Academic Press, New York, 1976.

4. R. Steinberg, Endomorphisms of linear algebraic groups, Mem. Amer. Math. Soc., no. 80, Amer Math. Soc., Providence, R. I., 1968.

5. , Lectures on Chevalley groups, Yale University notes, 1968.

Department of Mathematics and Statistics, University of Massachusetts, Amherst, MasSACHUSETTS 01003 (Current address)

Department of Mathematics, University of Virginia, Charlottesville, Virginia 22903 